(Aus dem Laboratorium der II. med. Klinik der Charité zu Berlin [Direktor: Geheimrat Kraus].)

\title{
Ein Beitrag zur Gefäßsyphilis des Gehirns.
}

\author{
Von \\ cand. med. Lucie Freund. \\ Mit 13 Textabbildungen.
}

(Eingegangen am 25. Januar 1921.)

Die Beteiligung der Hirngefäße an der Syphilis verschiedenster Form ist, wie lange bekannt, eine außerordentlich häufige, wenn nicht regelmäßige Erscheinung. Schon im sekundären Stadium der Lues können sich charakteristische Veränderungen ausbilden. Von $\mathrm{Naunyn}^{\mathbf{1}}$ ) wurde eindringlich darauf hingewiesen, daß am häufigsten bereits im ersten Jahre nach der Infektion eine syphilitische Erkrankung der Hirnarterien auftreten kann, und erst neuerdings wieder ist sie von Pirilä ${ }^{2}$ ) als eine bis zur Obliteration führende Endothelwucherung und lebhafte lymphocytäre Infiltration beschrieben worden. Im weiteren Verlaufe der Erkrankung, von den meisten Autoren als typisch für das Tertiärstadium angesehen, finden wir in den Hirngefäßen wie an denen des übrigen Körpers verschiedenste Arten pathologischer Veränderungen, wie Intimaproliferation, Aneurysmabildung, Infiltration der Adventitia u. a.

Mehrfach ist im Laufe der letzten Jahrzehnte der Versuch gemacht worden, die eine oder andere Form als eine wenigstens pathologisch, wenn nicht klinisch abgrenzbare Gruppe zu rechtfertigen. So hat He ub-

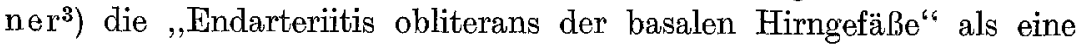
eigene Krankheitsform aufgestellt, während $\mathrm{Niss}^{4}$ ) und später Alzheimer ${ }^{5}$ ) die „Endarteriitis syphilitica der kleinen Hirnrindengefäße ${ }^{c_{6}}$

1) Zur Therapie und Prognose der syphilitischen Erkrankungen des Nervensystems, 1888.

2) Arbeiten aus dem Pathologischen Institut der Universität Helsingfors, Neue Folge 2, 1 u. 2. 1919.

3) Vogel, Leipzig 1874.

4) XXXIV. Versammlung südwestdeutscher Irrenärzte, Karlsruhe Nov. 1903.

5) Histologische und histopathologische Arbeiten über die Großhirnrinde I. 1904; Jahresversammlung des Deutschen Vereins für Psychiatrie, Dresden. April 1905 ; s. a. S pielmeyer, Alzheimers Lebenswerk, Zeitschr. f. d. ges. Neurol. u. Psychiatr. 1916. 
als eine Sondergruppe betrachtet haben, die sie den metasyphilitischen Erkrankungen zurechnen zu sollen glaubten.

Auffallend war allerdings, daß sich in der Folgezeit trotz der darauf gerichteten Aufmerksamkeit so außerordentlich spärlich reine Fälle von Endarteriitis syphilitica der kleinen Hirngefäße gefunden haben, während eine nicht unbedeutende Anzahl von Fällen einer ausgesprochenen Endart. syph. der kleinen Hirngefäße kombiniert mit andersartigen luetischen oder metaluetischen Erkrankungen bekannt wurde. So hat neuerdings $\mathrm{Ja}_{\mathrm{k} o \mathrm{~b}^{1}}$ ) auf ,die innige Verwandtschaft entzündlicher und nichtentzündlicher Formen der Syphilis im Zentralnervensystem" in einer größeren Zusammenfassung hingewiesen, in der er 13 Fälle von Endart. syph. der kleinen Hirnrindengefäße publiziert; bei diesen war in 7 Fällen die proliferative Gefäßerkrankung mit paralytischen resp. paralyseähnlichen Zustandsbildern kombiniert, und zwar fanden sich in 5 Fällen syphilitische Meningitiden, in 2 Fällen leicht infiltrative Erscheinungen an den Gefäßen, während drei Fälle paralytische Rindenveränderungen, in 1 Falle ohne Schichtstörungen, in 2 anderen mit starken architektonischen Verwerfungen und Rindenverschmälerungen aufwiesen.

Trotzdem scheinen einige pathologische Befunde dafür zu sprechen, daß die ausgesprochene Endart. syph. der kleinen Hirnrindengefäße, wenigstens der Gefäßsyphilis gegenüber, eine Sonderstellung einnehmen kann. Denn die systematische Spirochätenforschung im Gewebe hat gezeigt, daß die Spirillen bei den typisch endarteriitischen Formen in einem auffallend großen Prozentsatz der untersuchten Fälle fehlen, während sie sonst bei der Gefäßsyphilis einen regelmäßigen Befund darstellen.

Utberhaupt haben ja die neueren Untersuchungen über die Spirochäten im Gehirn und ihr Verhältnis zu den Gefäßen, wie sie am konsequentesten von $\mathrm{Jahnel}^{2}$ ) und in letzter Zeit vor allem von Haupt$\operatorname{man} n^{3}$ ) durehgeführt wurden, die Bedeutung der Gefäßgrenzen und ihrer biologischen Scheidewände gegenüber dem ektodermalen Gewebe wieder in den Vordergrund des Interesses gestellt und abermals die Frage nach einer neuen Formulierung des Lues-Metaluesprinzipes aufgeworfen.

Es wird also von diesem Gesichtspunkte aus zu erwägen sein, ob sich überhaupt eine scharfe Trennung der verschiedenen Formen der Gefäßsyphilis rechtfertigen läßt oder ob nicht vielmehr diese beiden ineinander übergehen können resp. verschiedene Stadien desselben Prozesses darstellen. Diese Anschauung vertritt $\mathrm{Benda}^{4}$ ) vom allgemeinen

1) Zeitschr. f. d. ges. Neurol. u. Psychiatr. 49. 1920.

2) Ebenda. 42. 1918.

3) Ebenda. 47. 1920.

4) Syphilitische Gefäßprozesse im Aschoffsehen Lehrbuch 1919; Die Syphilis des Gefäßsystems, Handbuch der Geschlechtskrankheiten. Finger. Wien 1912. 
pathologischen Standpunkt aus, indem er meint, es gäbe nur eine syphilitische Arteriitis, die syphilitische Periarteriitis Baumgartens $\mathbf{s}^{\mathbf{1}}$. Alle andern seien nur verschiedene Entwicklungsstadien, verschiedene Grade der Intensität und Extensität desselben Prozesses. Die Meinung Ba umgartens bestände auch zu Recht, daß der Befund dieser eigenartigen Intimaproliferation beim Fehlen anderer entsprechender Ätiologie den Wahrscheinlichkeitsschluß auf Syphilis zuließe. Ganz im Gegensatz zu diesen Anschauungen steht Heubners Formulierung der ,Endarteriitis obliterans der basalen Hirngefäße "als eines selbständig auftretenden Krankheitsbildes mit starker, bis zur Obliteration führender Intimawucherung basaler, makroskopisch veränderter Hirngefäße.

Von anderen Gesichtspunkten ausgehend kamen Nissl und Alzheimer zu einer besonderen Abgrenzung dieses pathologischen Befundes an den kleinen Rindengefäßen, indem sie diejenigen Gefäßerkrankungen, bei denen es vorzugsweise zu einer Proliferation, einer enormen Sprossung und zur Bildung vieler Lumina und neuer KapilIaren kommt, die zellige Exsudation aber fehlt, die ferner das Erhaltensein der gliösen Grenzhäute und eine geringere Affektion des Nervengewebes aufweisen, als metaluetische Gefäßerkrankung der Hirnrindengefäße beschrieben.

Im folgenden soll zunächst ein Fall als Ausgangspunkt der Diskussion dienen, bei dem sich verschiedenartige Formen der Gefäßsyphilis des Zentralnervensystems vereint finden, von der eigentlichen Endarteriitis obliterans Heubners über die Endarteriitis der kleinen Hirnrindengefäße bis zur ausgesprochenen Aneurysmabildung der Art. basilaris, kombiniert mit einer Tabes dorsalis; also eine Beteiligung der Gefäße jedes Kalibers von den Kapillaren, Präkapillaren an bis zur Art. basilaris an dem luetischen Prozeß unter mannigfachen Reaktionserscheinungen im Anschluß an eine entzündlich-degenerative Erkrankung des Rückenmarks.

Krankengeschichte.

Patient P., 39 Jahre alt, Bildhauer, fand im November 1901 Aufnahme im Krankenhaus Moabit. Von dort wird angegeben: Pat. klagt seit Juli d. Js. über taubes Gefühl in den Beinen, Gürtelgefühl, Kribbeln in allen Gliedern, Beschwerden beim Gehen und Urinlassen.

Herz, Lunge ohne Besonderheiten.

Patellar-, Bauchdecken- und Cremasterreflexe fehlen.

Pupillen reagieren nicht. Rechte Pupille kleiner als die linke.

Romberg +. Motilität: Aktive Bewegungen in der unteren Extremität nicht normal. Gang spreizend, unsicher. Knie-Hackenversuch ausfahrend. Muskeltonus der unteren Extremität herabgesetzt.

Ataxie beim Gehen auf einer Linie und Rückwärtsgehen deutlich.

1) Arch. f. Heilk. 16. 1875; Virchows Archiv y3, 1878 u. \%6. 1879. 
Sensibilität: Berührungsempfindung an der unteren Extremität herabgesetzt, stellenweise erloschen, Leitung streckenweise verlangsamt. Spitz-stumpf, warm-kalt werden nicht deutlich untersohieden.

Diagnose: Tabes dorsalis.

Pat. blieb bis zum 19. IV. 1902 in dortiger Behandlung, wurde dann als gebessert entlassen.

Im Juni 1903 suchte Pat. wegen zunehmender Harn- und Stuhlgangstörung wiederum Aufnahme im Krankenhaus.

Die folgenden Jahre brachte Pat. mit kurzen Unter breehungen in verschiedenen Krankenhäusern und Heilanstalten zu, doch bietet die Krankengeschichte der weiteren Aufenthalte kein Interesse. Der Prozeß verlief langsam progredient,

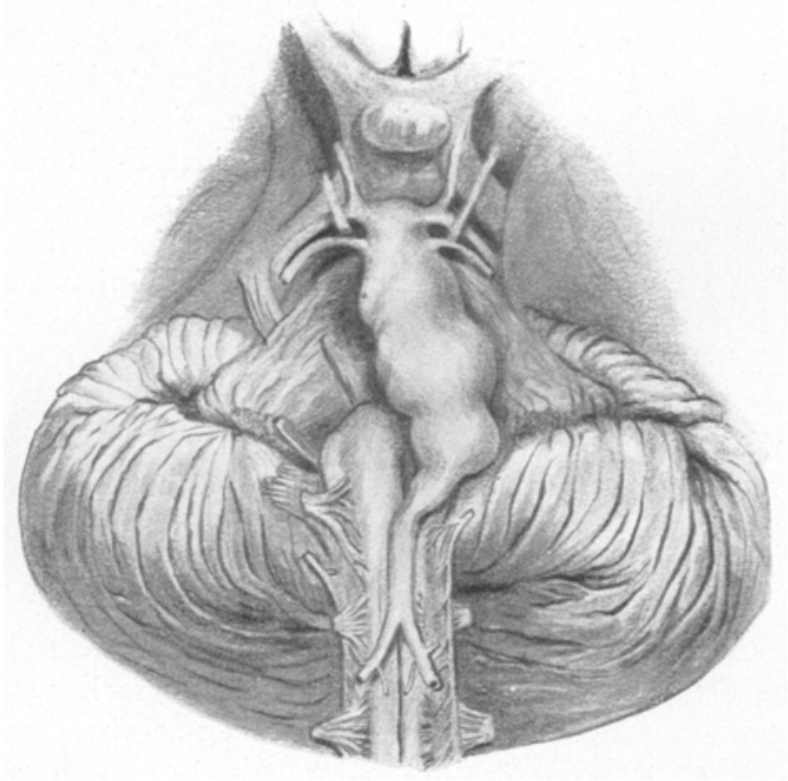

Abb. 1. Aneurysma der Art. basilaris.

besonders auffallende Erscheinungen kamen nicht zur Beobachtung. Dagegen scheint schon frünzeitig eine gewisse Cnstetigkeit bestanden zu haben, die zeitliche und örtliche Orientierung war zeitweise mangelhaft, ohne daß zunächst schwerere psychische Defekte in den Krankenhausbefunden vermerkt werden. Die Diagnose lautete stets: Tabes dorsalis, später in Verbindung mit progressiver Paxalyse.

Am 29. VII. 1912 wurde Pat. in das Städtische Siechenhaus Fröbelstraße eingeliefert. Pat. gibt an, am vorhergehenden Tage einen Schlaganfall mit rechtsseitiger Lähmung erlitten zu haben.

Befund: Rechte Seite paretisch, Reflexe nicht auslösbar, Sprache schleppend, Nachsprechen komplizierter Worte unmöglich. Zeitlich, örtlich desorientiert. Pat. ist sehr unruhig, drängt dauernd fort.

In den folgenden Tagen nimmt die rechtsseitige Lähmung weiter zu, die Sprache trägt ausgesprochen bulbären Charakter, aphasische Störungen fehlen. 
Unter zunehmender Benommenheit tritt infolge A te mlähmung am 1. VIII. 1912 der Tod ein.

Die Untersuchung des Gehirns ergab folgenden Befund:

Makroskopisch: Die Pia des Gehirns ist leicht getrübt, an einzelnen: Stellen verdickt, aber gut abziehbar.

Die Hinterstränge des Rückenmarks sind schmal und grau.

Eine ungefähr kirschkerngroße Stelle von grauroter Verfärbung, die über die Schnittfläche leicht hervorquillt, findet sich in dem Fußteil des: Pons und nimmt das Areal der linken Pyramidenbahn ein.

Die Art. basilaris ist fast in ihrem ganzen Verlaufe auf Kleinfingerdicke erweitert. Die diffuse, a neurysmatische Auftreibung beginnt ca. $1^{1 / 2}$ Quer. finger breit oberhalb ihres Ursprungs und erstreckt sich über das ganze Gefäß. bis zu der Teilung in die beiden Endarterien, die Artt. cerebri postt. Die Arterie ist nach links verlagert, überdeckt die linke Pyramide und Olive, überlagert auch. die Mitte, mehr noch die linke Hälfte des Pons und dellt ihn ein (s. Abb. 1). Auf dem Gefäßdurchschnitt erweist sich die Wand ungleichmäßig verdickt, an mehreren Stellen springt die Innenhaut beetartig in das Lumen vor; an einer solchen

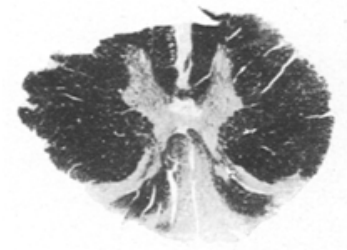

Abb. 2. Rückenmark. Spielmeyerfärbung.

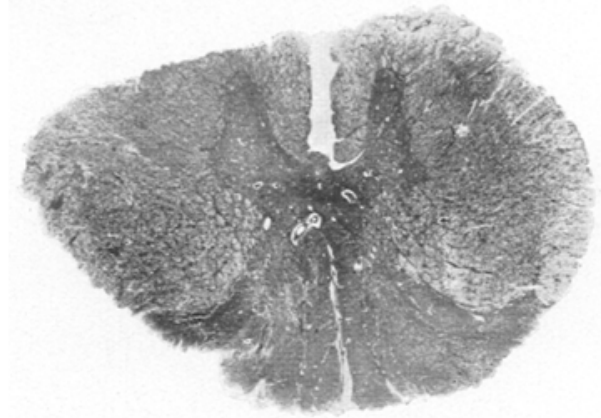

Abb. 3. Rückenmark. Gliawucherung in Hinter- und: Seitensträngen. Färbung nach Mann.

der Gehirnseite naheliegenden Stelle findet sich ein wandständiger, noch nicht. organisierter Thrombus. Auch andere Gefäße an der Konvexität des Gehirns sind' erweitert und thrombosiert.

Makroskopische Diagnose: Leichte Leptomeningitis, Tabes dorsalis, Basilaraneurysma mit sekundärem Erweichungsherdim Pons.

Mikros ko pis ch: Das Material wurde inAlkohol, Formalin und Gliabeize fixiert. und die Präparate nach den üblichen Methoden der Münchener Schule gefärbt.

Die Pia des Großhirns weist stellenweise eine mittlere Verdickung auf und ist herdförmig von mäßig starken Lymphocyteninfiltraten durchsetzt, ganz vereinzelt finden sich Plasmazellen. Die Pia des Rückenmarks ist etwas stärker mit: Lymphocyten infiltriert und zeigt keine erhebliche Verdickung.

Ein Querschnitt durch das Rückenmark (Abb. 2) zeigt in Markscheidenfärbung die totale Degeneration der Gollschen und eines Teiles der Burdachschen Hinterstränge, die einerseits völlig aufgehellt, andererseits merklich verschmälert sind. An den Pyramidenseitensträngen sind Aufhellungen mit dieser Färbung nicht wahrnehmbar. Eine Gliafärbung (Abb. 3) zeigt dagegen die diffuse, gliöse Wucherung, die nicht allein auf die Hinterstränge beschränkt bleibt, sondern sich auch in der ganzen Circumferenz der weißen Substanz als protoplasmatische Gliawucherung hervorhebt; besonders tritt der Untergang von nervöser Substanz. und die Gliawucherung im Gebiet beider Pyramidenseitenstränge deutlich zu Tage. Körnchenzellen und große Ausfälle fehlen. Die Kernfärbung eines Längsschnittes 
durch das Rückenmark zeigt deutlich die lymphocytäre und plasmazelluläre Infiltration z. B. in einem Gefäß der weißen Substanz aus dem Seitenstrang (Abb. 4) mit reaktiver Gliawucherung.

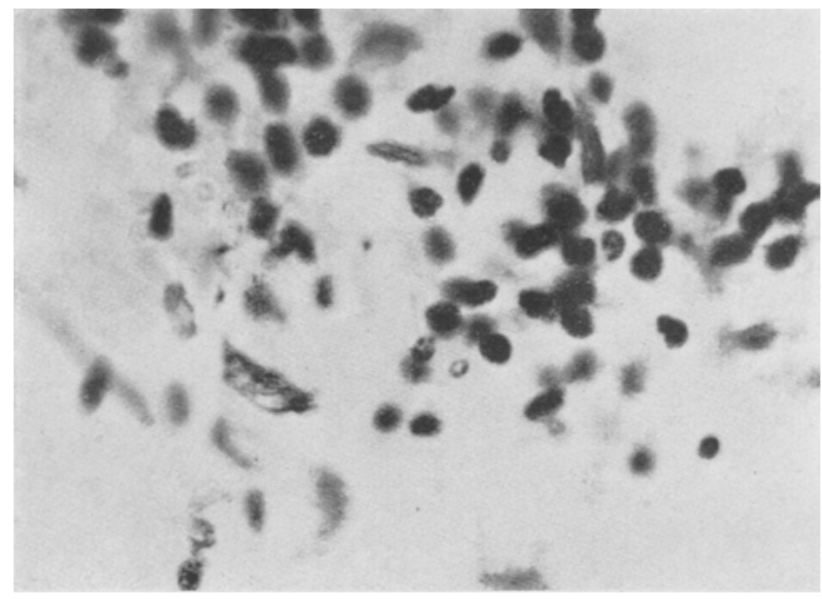

Abb. 4. Lymphozyten- und Plasmazelleninfiltration in und $1 \mathrm{~m}$ ein Gefäß der weißen Substanz im Seitenstrang des Rückenmarks. (Längsschnitt). Reaktive Gliawucherung. Färbung Tolluidivblau.

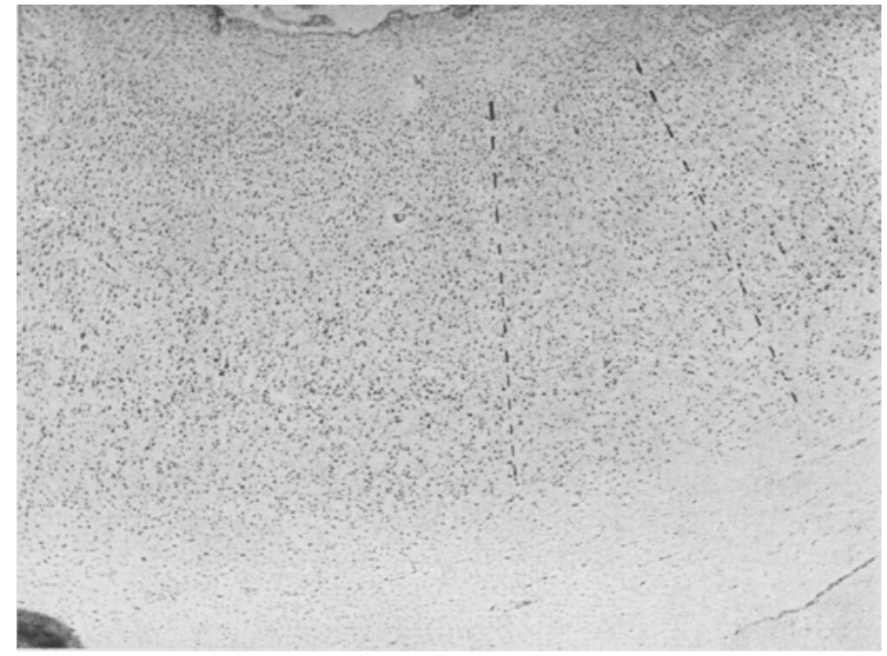

Abb. 5. Verödungsherd zwischen den beiden punktierten Linien; die Pyramidenschicht ist fast völlig ausgefallen, die anderen merklich reduziert. Färbung Tolluidinblau.

Im Großhirn ergibt eine Untersuchung der Marksubstanz keinerlei pathologische Veränderungen, weder in ihrer ektodermalen Struktur noch an ihren Gefäßen.

Dagegen weist die Rinde charakteristische Veränderungen auf: 
Die chronisch erkrankten Ganglienzellen haben dunkel gefärbte, pyknotische Kerne, ihre Fortsätze sind zum Teil geschlängelt und weithin sichtbar geworden. An anderen Stellen zeigen die Ganglienzellen folgende Veränderungen:

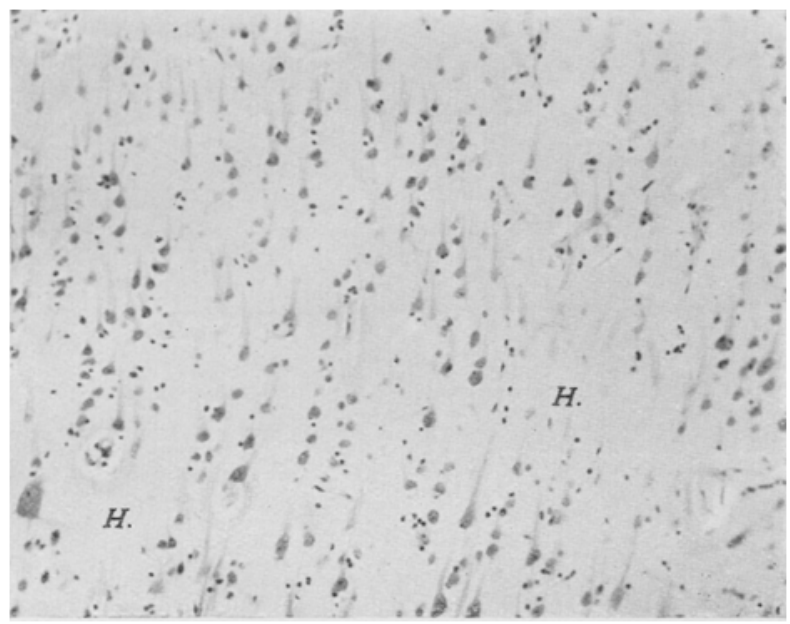

Abb. 6. Bei $H$ Verödungsherde in der dritten Schicht ohne Gliareaktion. Färbung Tolluidinblau.

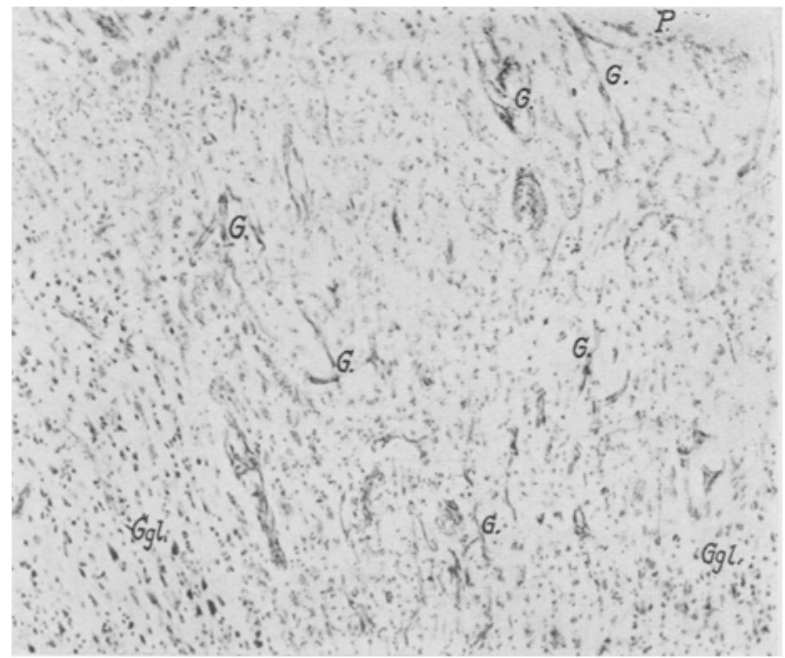

Abb. 7. Verödungsherd in der Rinde zwischen den guterhaltenen Ganglienzellreihen. Ggl. rechts und links. Oben Pia $(P)$. In dem verödeten Gebiete massenhaft neugebildete Gefäße, z. T. mit vielen Lumina, ohne Infiltrate. Keine Narbenschrumpfung. Färbung Tolluidinblau.

Der protoplasmatische Zelleib läbt keinerlei Nisslsche Schollen erkennen. Die Zellen haben teilweise ihre Färbbarkeit völlig verloren und sind nur noch als Schatten, ähnlich wie bei der arteriosklerotischen Verödung, sichtbar. Überall 
zeigt sich als Reaktion die starke Gliawucherung, ohne daß es zu einer Faserbildung kommt. Frei im Gewebe liegt eine Anzahl von Lymphocyten; Stäbchenzellen konnten nicht gefunden werden. Die Architektonik der Hirnrinde ist wenig gestört, nur vereinzelt findet man im Anschluß an erkrankte Gefäße Bezirke, in denen die Ganglienzellen schichtweise schlechter färbbar geworden sind (Abb. 5). Zwischen den beiden punktierten Linien sieht man auf Abb. 5, daß die Pyramidenschicht fast völlig ausgefallen ist, während die anderen Zonen merklich reduziert erscheinen. Die Abb. 6 bei starker Vergrößerung zeigt den völligen Zellschwund bei $H$ ohne Gliareaktion.

An anderen Stellen steht der GefäßwucherungsprozeB ganz in Vordergrund (Abb. 7). Die Rinde ist diffus durchsetzt von massenhaften neugebildeten oder

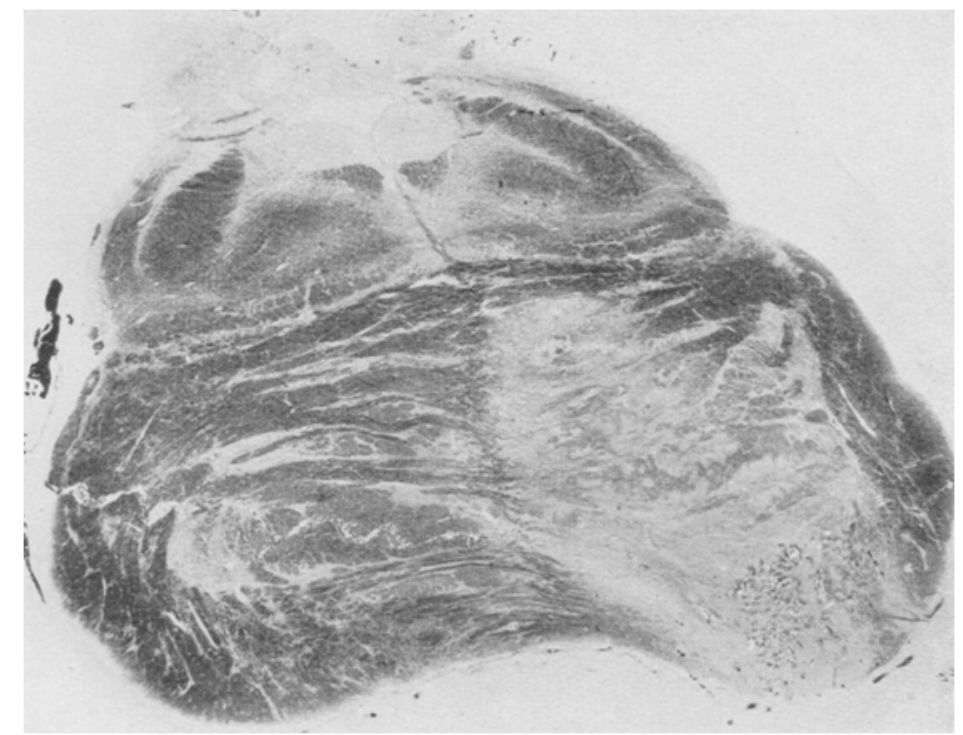

Abb. 8. Druckerweichung des Pons. Färbung Tolluidinblau.

gewucherten Kapillaren. Diesen fehlen jegliche Infiltrate. Das Nervengewebe ist leicht atrophisch, besonders Ganglienzellen sind reichlich zugrunde gegangen, dafür ist die Glia massig gewuchert, vor allem finden sich reichlich große, gemästete Gliaformen. Zu einer Strukturveränderung ist es nicht gekommen.

Abb. 8 gibt im Übersichtsbild den Erweichungsherd des Pons in seiner ganzen Ausdehnung wieder. Bei starker Vergrößerung sind bei Zellfärbung diffuse 'Blutungen frei im Gewebe deutlich erkennbar; im Zentrum des Herdes fehlt die Kernfärbung völlig, während der dem Herde unmittelbar anschließende Teil caudalwärts Quellung und Erweichung zeigt. Die Mannsche Färbung dieses Erweichungsherdes zeigt folgendes Bild: Die Achsenzylinder sind gequollen, gesehlängelt oder abgerissen; in den Markseheiden sieht man stellenweise Elzholzsche Körperchen, an anderen Stellen sind sie ganz unfärbbar geworden. Ferner kann man an vereinzelten Stellen Myeloklasten erkennen.

Von der thrombosierten Art. basilaris wurden an einer spindelförmig erweiterten Stelle nach der von Ranke angegebenen Modifikation Serienschnitte angefertigt und auf Elastica, Kerne und Silberfasern gefärbt. Der Thrombus selbst 


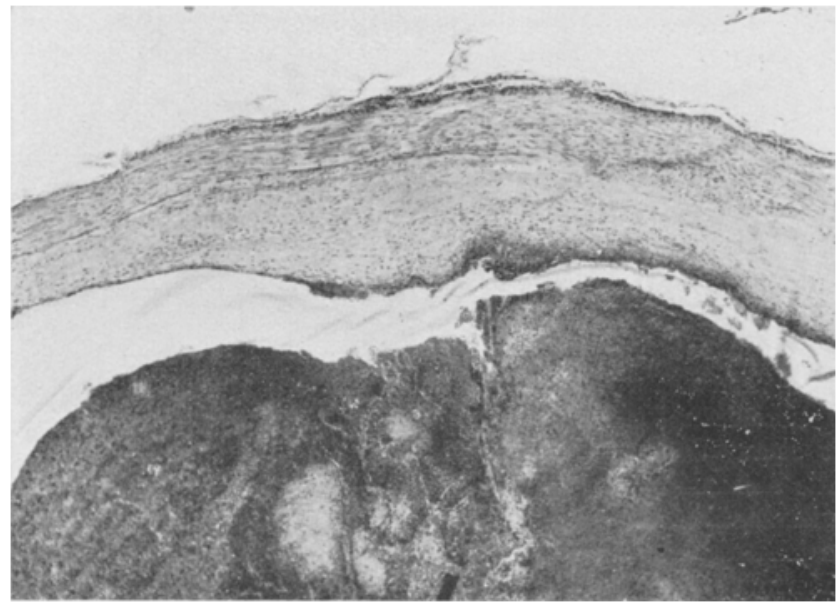

Abb. 9. Art. basilaris. Elastikafärbung.

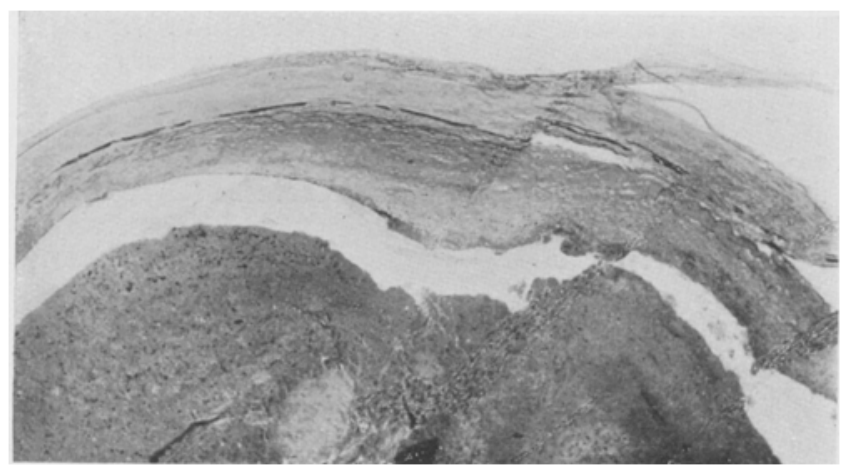

Abb. 10. Art. basilaris. Tannin-Silberfärbung.

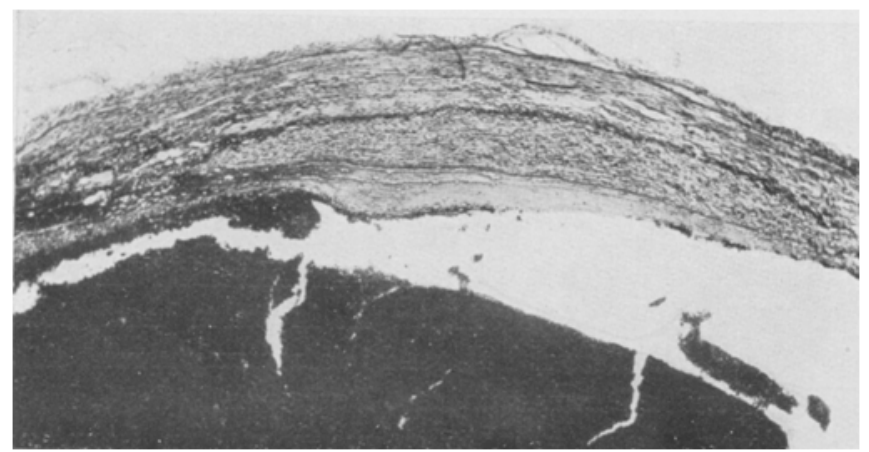

Abb. 11. Art. basilaris, van Giesonfärbung. 
bildet eine einheitliche Masse und neigt dazu, beim Schneiden herauszufallen. An der Gefäßwand, deren Ungleichmäßigkeit sofort ins Auge fällt, sieht man, soweit die Endothellage noch einschichtig ist, die Kerne der Endothelzellen gebläht und in das Lumen vorspringend, ihr Plasma hat eine intensive Färbung angenommen. An den meisten Stellen finden sich bereits mehrere En dothellagen; an vier sich etwa diagonal gegenüberliegenden Stellen ist es zu einer beetartigen, weit in das Lumen vorspringenden Wucherung gekommen, in deren Tiefe einzelne Kerne sich bereits zurückzubilden beginnen und wo das Auftreten von Körnchenzellen den Anfang regressiver Prozesse anzeigt, Zwischen diesen neugebildeten Endothelschichten treten feinste Elastikafasern auf (Abb. 9), während die ursprüngliche Elastica interna aufgesplittert, stellenweise eingerollt und auf ganze Strecken hin völlig verschwunden ist. Im Zusammenhang mit den geschilderten degenerativen Vorgängen der Intima, den Neubildungen elastischer Lamellen, ist es zur Wucherung versilberbaren mesodermalen Gewebes gekommen, das auch die Muscularis durchsetzt (Abb. 10). Die Muskelschicht der Media exscheint verschmälert und fettig degeneriert, auch zeigt sich stellenweise das Auftreten von Körnchenzellen. Auf den entzündlichen Charakter der Erkrankung weist die Kernfärbung der gleichen Stelle hin (Abb. 11), bei der man eine herdförmig verteilte, massige Infiltration der Adventitia mit Rundzellen wahrnimmt; in geringerem Grade ist eine herdförmige Durchsetzung der gewucherten Intima mit Rundzellen sichtbar.

Vor allem die kleinen Gefäße der Pia, aber auch die in die Rinde einstrahlenden Kapillaren und Präkapillaren, mit besonderer Bevorzugung des Frontallappens, zeigen weitgehende Veränderungen, die sich mit den Vorgängen in dem großen Gefäßrohr im wesentlichen decken. Das Auffälligste ist die e nor me Intimawucherung, die die Dicke der Gefäßwand um das Vier- bis Fünffache übertrifft. Auf dem Weigertpräparat einer Pialarterie (Abb. 12) erscheint die Elastica sehr stark geschlängelt, sie fehlt auf großen Strecken vollständig, und vereinzelt ist auch hier die Aufsplitterung der alten und gleichzeitig die Neubildung mehrerer Lamellen deutlich erkennbar. Die Kernfärbung der gleichen Stelle (Abb. 13) bringt die starke Rundzelleninfiltration in der Adventitia, eine schwächere auch in der Intima sowie in der Muscularis zum Ausdruck.

An zahlreichen Blöcken aus allen Teilen des Zentralnervensystems und des. Basilaraneurysmas, die nach Jahnel gefärbt waren, haben sich Spirochäten nicht nachweisen lassen.

Mikroskopische Diagnose: Es findet sich, begleitet von einer leichten Leptomeningitis des Großhirns, einer etwas stärkeren des Rückenmarks, eine typische Tabes dorsalis in Kombination mit einer Seitenstrangdegeneration. Ferner eine Endarteriitis syphilitica der kleinen Hirnrinden- und Pialgefä $B$ e, teilweise bis zur Obliteration führend mit Verödungsherden; ein Aneurysma der Art. basilaris mit Thrombose and sekundärem Erweichungsherd im EuBteil des Pons.

Nach dem klinischen Verlauf des geschilderten Falles hatte man sich die Vorstellung gebildet, $\mathrm{da} B$ sich im Anschluß an eine Tabes dorsalis eine Paralyse entwickelt hätte, die in einem paralytischen Anfalle zum Tode führte. Diese naheliegende Vermutung erweist sich jedoch nach dem vorliegenden Befunde als irrig. Da inner halb des Gehirns weder für Arteriosklerose Anhaltspunkte gefunden werden konnten, noch Gummibildungen aufzufinden waren, und auch differentialdiagnostisch 
für eine Paralyse nichts spricht, muß man den Prozeß im wesentlichen in einer herdförmig angeordneten, krankhaften Veränderung der Gefäße vorwiegend progressiven Charakters sehen, die zu einem fleckförmigen Ausfall

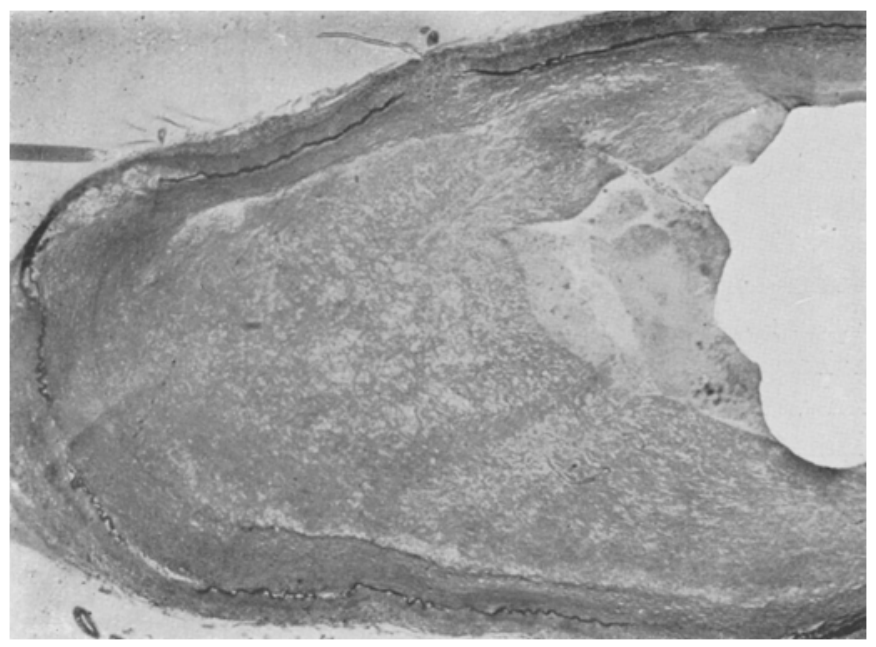

Abb. 12. Pialarterie. Elastikafärbung.

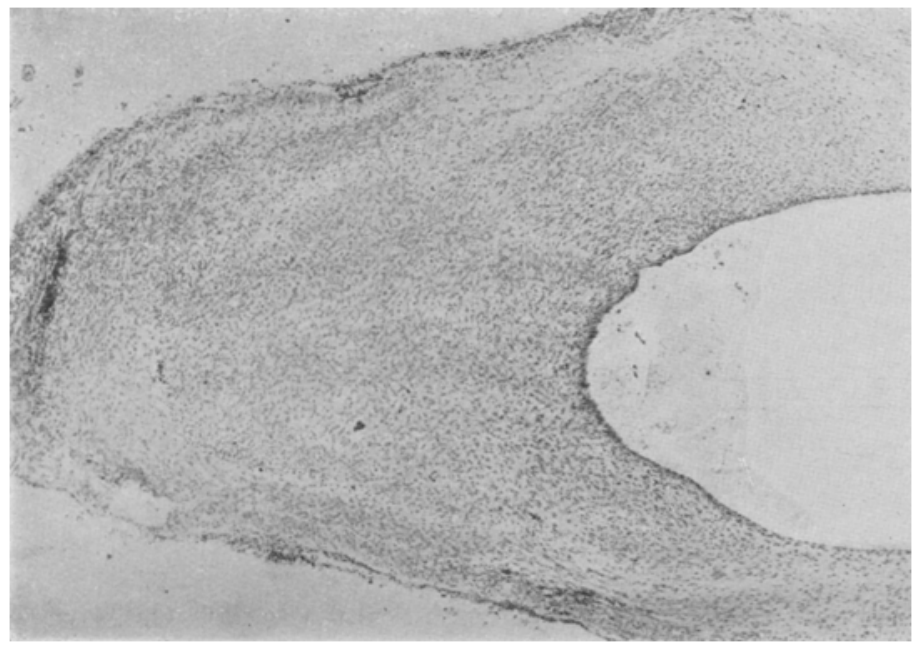

Abb, 13. Pialarterie. Färbung Tolluidinblau.

von Ganglienzellen geführt hat. Der proliferative Gefäßprozeß zeigt im wesentlichen die Kriterien der von Nissl und Alzheimer beschriebenen ,Endarteriitis syphilitica der kleinen Hirnrindengefäße", doch zeigen unsere Präparate den leicht entzündlichen Charakter der Ver- 
änderungen durch Auftreten von Rundzelleninfiltraten, den Alzhei mer für seine Fälle verneint. Ferner konnten die von Alzhei mer für charakteristisch angesehenen Stäbchenzellen nicht gefunden werden. Gewisse Eigentümlichkeiten unseres Falles erinnern auch an die von. He ubner beschriebene „Endarteriitis syphilitica obliterans der basalen Hirngefäße"; doch ist der Prozeß im vorliegenden Falle nicht auf die Gefäße mittleren Kalibers beschränkt geblieben, sondern hat auch die kleinen Arterien ergriffen. Als Prototyp der Erkrankung eines basalen Gefäßes mittleren Kalibers sahen wir die Veränderungen an der Art. basilaris, bei der gleichzeitig Intimawucherungen und degenerative Mediaerkrankung mit Aneurysmabildung bestehen. Als sekundäre Folge dieses Aneurysmas und seiner Thrombose ist der Erweichungsherd im Pons anzusehen, der klinisch apoplektiform entstanden ist.

Fassen wir unsere Befunde zusammen, so sehen wir, daß es sich in der Hauptsache um einen luetischen Gefäßprozeß handelt, der teils in das Gebiet der sog. Endart. syph. der kleinen Hirnrindengefäße, teils in das der Endart. syph. obliterans Heubners, teils in das einer Mediaerkrankung mit Aneurysmabildung fällt. Es bleibt nun zu überlegen, wie man sich das Verhältnis dieser verschiedenartigen pathologischen Befunde zueinander zu denken hat, ob man zu der Annahme berechtigt ist, daß es sich hier um verschiedene Stadien ein und desselben Prozesses handelt, oder ob man ein zufälliges Zusammentreffen dieser mannigfachen Gefäßerkrankungen annehmen will.

Was zunächst den Zusammenhang zwischen der sog. Endart. der kleinen Rindengefäße und der Heubnerschen Form an den basalen Gefäßen anbetrifft, so hält Alzheimer selbst ihn für einen engen; anders steht es mit der Beziehung zwischen Intima- und Mediaerkrankung.

Daher soll zunächst die Histopathogenese der durch Lues bedingten Veränderungen an den kleinen Hirngefäßen uns Veranlassung geben, das Zustandekommen der Intimaproliferation und die Möglichkeit der Kombination mit Aneurysmabildung an einem mittleren Hirngefäß zu verstehen.

Die grundlegende Arbeit Heubners aus dem Jahre 1874 über die Endarteriitis syphilitica der basalen Hirngefäße, der das Charakteristikum dieses Prozesses, welcher primär von der Gefäßinnenhaut ausgeht, in der auffallend starken Intimawucherung, dem Fehlen regressiver Metamorphosen, in der mehr oder weniger isolierten Herdbeteiligung bei Bevorzugung der kleinen und mittleren Arterien (im Gegensatz zur Arteriosklerose) fand, riefen bald lebhaften Widerspruch hervor. Es folgten jahrelang Diskussionen über den Ort der Entstehung der Veränderungen. Zunächst wurde der spezifische Charakter des 


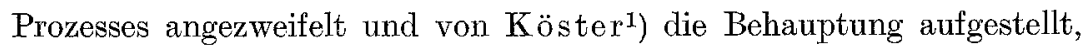
daß die Lues zwar eine Disposition, aber kein Spezificum für die beschriebenen Veränderungen bilde, und die Identität mit der durch Ligaturen und andere Schädlichkeiten bedingten Endarteriitis obliterans nachgewiesen. Dagegen stellte Baumgarten 1875 den spezifischen Charakter der Gefäßveränderungen fest. Er verlegte den Beginn der Erkrankung in die Adventitia, dort ausgehend von gummösen Herden, einer Periarteriitis gummosa, die eine sekundäre Intimawucher ung hervorrufen könne. Dieser Ansicht schlossen sich später namhafte Autoren an. Rum pf ${ }^{2}$ ) sieht dagegen das Primäre in einer Erkrankung des Kapillarsystems der Media und einer von dort ausgehenden Verbreitung auf dem Wege der Vasa vas., entweder in das Gebiet der Intima oder in das der Adventitia, wobei die Media an dem krankhaften Prozeß wenig beteiligt ist. So löst er den Widerspruch zwischen $\mathrm{Heub-}$ ner und Baumgarten. Auch Nonne und $\mathrm{Luce}^{3}$ ) vertreten die Meinung, daß eine durch den spezifischen Reiz produzierte Entzündung der Gefäßkapillaren zu einer entzündlichen Wanderkrankung führe, .zwar in erster Linie von Media und Adventitia; sekundär aber erkranke die Intima, und man habe das Bild der Heubnerschen Endarteriitis mit Verengerung und Obliteration des Lumens vor sich. Andere Autoren, wie Oppenheim ${ }^{4}$ ), auch Darier ${ }^{5}$, nehmen dagegen ein gleichzeitiges Befallensein aller drei Häute an und sprechen von einer Panarteriitis. Am einheitlichsten beurteilt Benda die Intimaproliferation. Er bestreitet den entzündlichen Charakter derselben und nimmt an, daß die Intima bei jeder Schädigung der Gefäßwand, gleichviel in welcher Schicht, kompensatorisch in Wucherung gerate.

Nach den Erfahrungen spricht vieles dafür, daß der Prozeß seinen Ursprung von den Vasa vasorum nimmt. Denn die Krankheitsursache wird ja durch die Schädigungen gegeben, die die Spirochäten setzen. und da ist es nach biologisehen Prinzipien sehr einleuchtend, daß sie sich die Orte aussuchen, an denen sie die besten Existenzmöglichleiten finden. Die Gehirnarterien haben, wie schon Köster nachwies, eine besondere Disposition zu luetischen Affektionen. da sie bis in ihre feineren Verzweigungen mit Nährgefäßen versehen sind.

Die verschiedenen Gefäßschichten antworten nun auf den ihnen durch ihre Nährgefäße zugetragenen Reiz auf eine spezifische Weise. In der Intima entwickelt sich die stärkste Reaktion, die in einer beetartigen Wucherung ihres Endothels als typisch endarteriitisch-luetischer

1) Berl. klin. Wochenschr. 31. 1876.

2) Die syphilitischen Erkrankungen des Nervensystems. Wiesbaden 1887.

3) Handbuch der pathologischen Anatomie des Nervensystems. Berlin 1904.

4) Zur Kenntnis der syphilitischen Erkrankungen des Zentralnervensystems. Berlin 1890.

5) De l'artérite syphilitique. Paris 1904. 
Prozeß gekennzeichnet ist. Die Media, die in den kleinen Hirngefäßen eine sehr geringe Rolle spielt, ist an der Erkrankung auffallend wenig beteiligt. Dagegen sehen wir sie in mittleren und größeren Gefäßen in den Krankheitsproze $\beta$ hineinbezogen. Im muskulären Anteil finden sich Degenerationserscheinungen, vor allem fettige Entartung und Zerfall der Muskelfasern; die elas' ischen Elemente sind dissoziiert. aufgerollt, fehlen streckenweise ganz und zeigen an anderen Stellen lamellöse Neubildungen [Kerppola1)]. In der Adventitia findet sich häufig, doch nicht regelmäßig, eine herdförmige Rundzelleninfiltration.

Die Beziehung der Endart. syph. der Hirnrindengefäße zu der Gefäßsyphilis des übrigen Körpers müssen wir in einer Anpassung des Krankheitsprozesses an den gegebenen histologischen Bau der Hirngefäße sehen. In anderen Arterien des Körpers entwickelt sich im allgemeinen eine Mesarteriitis, während in den Arterien des nervösen Parenchyms eine Endarteriitis entsteht, die sämtliche Kriterien des von Heubner und Alzheimer aufgestellten Krankheitsbildes mit Ausnahme der Entzündung aufweist.

Im vorliegenden Falle findet sich außer dem eben besprochenen. häufigen Befunde eine relativ seltene Form syphilitischer Erkrankungen der Hirngefäßé, ein A neurys ma der Art. basilaris. Über die Kombination dieser beiden luetischen Gefäßprozesse ist wenig bekannt; es finden sich in der Literatur die Angaben von Chvostek und Weichselbaum² aus dem Jahre 1877 über ,herdweise syphilitische Endarteriitis mit multipler Aneurysmabildung", in der ausgeführt wird, daß der Ausgangspunkt der Veränderungen in der Proliferation der Intima bei zunächst intakter Elastica zu suchen sei; später erfolgen Einrisse, und durch diese hervorgerufen tritt Erweichung der Media ein, die dann zur Ausbildung der Aneurysmawandung führt. Benda ${ }^{3}$ ), der sich in seinem Korreferat in der Deutschen Pathologischen Gesellschaft 1903 eingehend mit dieser Frage befaßt hat, kommt zu dem Ergebnis, daß endarteriitische Wucherung und Aneurysmabildung einander entgegengesetzte, sich nicht begünstigende Prozesse seien, mit der Begründung, ,daß die produktiven Vorgänge in der Intima so stark in den Vordergrund treten, daß eine Verdickung der Wand bis zur Obliteration erfolgt, die durch häufig dazukommende Thrombose vervollständigt wird, so daß an den dieser Art erkrankten Stellen die Möglichkeit einer Aneurysmabildung ausgeschlossen erscheint ${ }^{\text {"c }}$. Baumgarten und Eppinger ${ }^{4}$ ) vertreten die gleiche Anschauung, während

1) Arbeiten aus dem Pathologischen Institut der Universität Helsingfors. Neue Folge 2, 1. u. 2. 1919.

2) Allg. Wien. med. Zeitung 1877 .

3) Korreferat aus den Verhandlungen der Deutschen Pathologischen Gesellschaft. 6. Tagung. Kassel 1903.

$\left.{ }^{4}\right)$ Arch. f. klin. Chir. 35, Suppl, 1887. 
von französichen Autoren [Die ulafo $\mathrm{y}^{1}$ ), S pillma $n n^{2}$ ), Thibierge ${ }^{3}$ )], vor allem von Lancerea $x^{4}$ ), darauf hingewiesen wird, daß die Arteriitis der Hirnarterien besonders zur Bildung von Aneurysmen geeignet sei, eine Ansicht, die auch deutseherseits von Chvostek und Weichselbaum, Abra $\mathrm{mow}^{5}$ ) und P. Müller ${ }^{6}$ ) vertreten wird. In gleichem Sinne zeigt eine Arbeit aus neuester Zeit von Kerp pola das Nebeneinander von endarteriitischer Wucherung und aneurysmatischer Erweiterung an den Basalarterien des Gehirns und geht an Hand von 13 Fällen ausführlich auf die Histologie dieser Erscheinungen ein.

Es ist aus der Literatur ersichtlich, daß aneurysmatische Erweiterungen an den Gefäßen des Zentralnervensystems ihren häufigsten Sitz an der Art. basilaris haben. Be nda räumt der Basilararterie an Häufigkeit sogar den dritten Platz unter allen Körperarterien ein. Bereits 1866 weist Lebert ${ }^{7}$ ) auf die praedisponierende Lage des Circulus - arteriosus Willisii zur Aneurysmabildung hin. Für ihre Ätiologie interessant sind die Versuche von Vanzetti ${ }^{8}$ ), der durch Utberimpfung spirochätenhaltigen Materials aneurysmatische Gefäßveränderungen erzeugte.

Will man nicht annehmen, daß ein Zufall die vorliegende Kombination von Endarteriitis und Aneurysma bedingt hat, so muß man sich wohl das Verhältnis der beiden Gefäßerkrankungen in Übereinstimmung mit Kerppolas Ausführungen folgendermaßen vorstellen:

Durch die auf dem Wege der Vasa vas. zugefübrte Schädlichkeit entarten primär die Elemente der Media, indem die Muskelfasern verfetten, die ganze Schicht verschmälert wird und schließlich auch die widerstandsfähigeren elastischen Membranen in Mitleidenschaft gezogen werden, d. h. sie werden auseinandergezerrt, gedehnt und zerrissen, und es entsteht eine bindegewebige Narbe; kurz es entwickelt sich das oben beschriebene Bild. An solchen Stellen, an denen Muskelfasern und elastisches Gewebe zugrunde gehen bzw. durch funktionell minderwertiges Bindegewebe ersetzt werden, sehen wir, worauf Kerppola ausdrücklich hinweist, eine reaktive Intimaproliferation einsetzen, die die stärksten Grade erreichen, also zu einer Verlegung des Gefäßlumens führen kann. Stellenweise sieht man auch an solch geschädigtem Gefäß eine Infiltration der Adventitia mit Runḋzellen auftreten.

1) Gaz. hebdom. des sciences méd. de Bordeaux 1892.

2) Annalen d. Dermat. \%, 11, 12. 1886.

3). Gaz. des hôp. 62. 1889.

4) Gaz. hebdom. des sciences méd. de Bordeaux 1882.

5) Zieglers Beiträge z. allg. Pathol. u. pathol. Anat. 26. 1899.

6) Festschrift zur Feier des 50 jährigen Bestehens des Stadtkrankenhauses Dresden-Friedrichsfelde. Dresden 1899.

7) Berrl. klin. Wochenschr. $20 \mathrm{ff} .1866$.

8) Arch. per le scienze med. 1911. 
Es kommt also im wesentlichen beim Zustandekommen des Krankheitsbildes darauf an, ob die Alteration der Media überwiegt - dann entsteht ein Aneurysma - oder ob die kompensatorische Intimawucherung so kräftig ist, daß die veränderte Gefäßwand dem Blutdrucke einen normalen Widerstand entgegensetzen kann - dann entsteht die sog. Endarteriitis mit all ihren Folgeerscheinungen.

Die relative Seltenheit aneurysmatischer Veränderungen an den Hirngefäßen im Gegensatz zu dem häufigen Befunde proliferativer Intimaprozesse daselbst gibt zu der Erwägung Veranlassung, daß hier für das Zustandekommen des Endeffektes, eben der Erweiterung des Gefäßes, das Zusammenwirken mehrerer Faktoren eine gewisse Rolle spielen muß. Auch muß, entgegen Kerppola, ausdrücklich auf den Unterschied in der Lokalisation beider GefäBprozesse hingewiesen werden, denn die Intimawucherung ist allgemein im Gebiete der kleinen Arterien anzutreffen, während die Aneurysmabildung meistens nur an größeren und mittlerèn Gefäßen Platz greift.

Es bestünde die Möglichkeit einer erhöhten Disposition zu atheromatösen Veränderungen, die an einem Gefäß mittleren Kalibers ja nicht a priori auszuschließen sind, einem Faktor, dem Kerppola die größte Bedeutung beimißt.

Dem steht entgegen, daß nach Heiberg') Fett- und Kalkdegenerationen im Gefäße selten an den syphilitisch veränderten Stellen auftreten. Das gleiche Verhalten fand $\mathrm{Faber}^{2}$ ) an 5 Aorten. Vielleicht liegt auch eine besonders starke Ansiedlung von Spirochäten oder eine akute Ausbreitung des Prozesses nach dem Typus der Darierschen Panarteriitis (Benda) vor. Eventuell wäre auch mit einer Schädigung der Wand durch mechanische Insulte zu rechnen (Lebert), die einen locus minoris resistentiae bedingt haben. Jedenfalls ist eine Entscheidung über das auslösende Moment nach den histologischen Untersuchungsmethoden zur Zeit nicht möglich. Auch müssen die Schlüsse, die wir aus den Beobachtungen ziehen, mit aller Reserve gemacht werden, da das vorliegende Material bei weitem nicht ausreichend ist, um daraus allgemein gültige Regeln für die Genese der beschriebenen kombinierten Gefäßerkrankungen syphilitischer Ätiologie abzuleiten.

Das Auftreten endothelialer Wucherungen und ihre Beziehungen zum Nervengewebe können wir an Abbildung 7 verfolgen, die an einer umschriebenen Stelle an sonst intaktem Rindengewebe eine bündelförmige Anordnung neugebildeter Gefäße ohne Infiltration zeigt. Die Ganglienzellen gehen infolge der durch Obliteration bedingten mangelhaften bzw. fehlenden Ernährung allmählich zugrunde, und zwar werden die höchstdifferenzierten Zellen zuerst von der Ernährungsstörung

1) Nord. med. Arch. 10.

2) Die Arteriosklerose. Jena 1912. 
betroffen, während die Gliaelemente nicht von dem ProzeB ergriffen sind. Der Prozeß verläuft so milde, daß Zeit genug vorhanden ist, $\mathrm{da} \beta$ in dem verödeten Bezirke die Glia z. T. sogar massig wuchert, so daß es nicht zu einer Retraktion des betreffenden Gewebes kommt, sondern daß eine Substitution eintritt. Da in unserem Falle aus dem Vergleich mit anderen Präparaten im vorliegenden Stadium keine direkte Spirochätenschädigung des nervösen Gewebes nachweisbar war, muß man zu der Annahme neigen, daß die Gefäßneubildung hier das Primäre, die Veränderung des nervösen Parenchyms das Sekundäre ist; allerdings darf dabei nicht außer acht gelassen werden, daß beide Prozesse Hand in Hand gehen, so daß einerseits durch die Wucherung der Gefäßinnenhaut die Parenchymzellen aus trophischen Gründen geschädigt werden, andererseits aber der erhöhte Zellzerfall wiederum Veranlassung zur Neubildung und zum Einwachsen der Gefäßsprossen gibt. Die Wucherung der Gefäße geschieht nicht im Sinne einer mesodermalen Narbenbildung, sondern ist eher einer angiomatösen Wucherung vergleichbar. In der Literatur finden wir ähnliche Angaben bei We ndeler ${ }^{1}$ ), der an seinem Fall endarteriitischer Lues der Hirnarterien vor allem die Tendenz der Kapillaren zu Sproßbildungen hervorhebt und angibt, bei seinem Material in einem Falle bis zu 45 neugebildeter Blutbahnen gefunden zu haben. Einen entsprechenden, wenn auch in dieser Richtung nicht so ausgesprochenen Befund enthält die Arbeit von Sage ${ }^{2}$ ).

Von Wichtigkeit und Interesse erscheint das Verhalten der Spirochäten in den so veränderten Gefäßen. Es wurde schon eingangs darauf hingewiesen, daß ein positiver Spirochätenbefund bei der gewöhnlichen Gefäßlues ein regelmäßiges Vorkommnis sei. In unserem Falle konnte trotz eifrigen Suchens kein Erreger in den erkrankten Blutbahnen oder irgendwo im Gewebe gefunden werden. Auch sind mir aus der Literatur keine Fälle endarteriitischer Hirnlues der kleinen Rindengefäße mit positivem Spirochätenbefund bekannt geworden; Benda berichtet über einen Fall in seiner großen Zusammenfassung im Aschoff schen Lehrbuch, bei dem er im adventitiellen Infiltrat von thrombosierten, syphilitisch veränderten Hirngefäßen mit tle rer Größe außergewöhnlich dicht gelagerte Spirochäten auffand. Eine ähnliche Lokalisation weist ein Befund von Strassmann auf ${ }^{3}$ ), bei dem sich die Spirochäten am zahlreichsten in der Adventitia und in den "Lymphscheiden" fanden. Doch stimmen alle Autoren darin überein, daß der Befund von Parasiten bei sog. endarteriitischen Prozessen ein seltener ist.

Es wurden bei Besprechung der Genese der luetischen Endothel-

1) Dtsch. Arch. f, klin. Med. 55. 1895.

2) Zeitschr. f. d. ges. Neurol. u. Psychiatr. 3. 1910.

3) Zieglers Beiträge z. allg. Pathol. u. pathol. Anat. 49. 1910. 
proliferation die mannigfachen, weit auseinandergehenden Theorien besprochen, die in dieser Richtung bestehen. So werden auch von histobiologischer Seite sehr differente Angaben über den Infektionsweg der Spirochäten in den Gefäßen gemacht. Ranke $\mathrm{k}^{1}$ ) hat eine primäre Einwirkung des syphilitischen Virus auf die Intima angenommen, den Intimaprozeß also als den ersten angesehen, wie es $\nabla$ ers é ${ }^{2}$ ) für die Venen beschreibt, während andere Autoren den Angriffspunkt aus den schon oben angeführten Gründen in die Vasa vas. verlegen und den adventitiellen ProzeB für den. frühesten halten (Benda, Strassmann). Pirilä glaubt, daß von dort aus eine Vermehrung und einWeiterwandern längs der Lymphspalten und Gefäße in der Adventitia statthat. Ein dritter Teil der Autoren vertritt schließlich die Ansicht, daß alle drei Sehichten gleichzeitig befallen werden. In der aus neuester Zeit stammenden Arbeit von $\mathrm{Ha}$ u ptma n $\mathrm{n}$ über „Die Spirochäten und Hirnrindengefäße bei Paralyse" geht der Verfasser in ausführlicher Weise auf das biologische Verhalten der Krankheitserreger, ihre Beziehungen zu den, Gefäßen und zu dem nervösen Parenchym ein. Für die bakterioskopische Untersuchung zieht er die Folgerungen aus den Nisslschen Anschauungen, der die Limitans gliae als biologischen Trennungsfaktor zwischen luetischen und metaluetischen Erkrankungen aufgestellt hat, und gibt der Erwägung Raum, daß vielleicht Verschiedenheiten in der Spirochätendurchsetzung der Gefäße maßgebend für den Krankheitsverlauf sein könnten. Ferner führt Ha uptmann in seinen Gedankengängen aus, daß eine besondere Affinität der Spirochäten zu der unmittelbaren Umgebung der Gefäße nicht in mechanischen Faktoren des Gewebes zu sehen sei, sondern vielleicht durch die biologischen Eigensehaften der Spirochäten erklärt werden müßte.

Wie wir schon oben kurz andeuteten, scheint manches zu der Annahme zu berechtigen, daß die Spirochäten aktiv diejenigen Stellen aufsuchen, an denen ihnen die besten Existenzmöglichkeiten geboten werden. Aus ihrem Charakter als Lymphparasiten wird erklärlich, daß sie die den Gefäßen unmittelbar anliegenden adventitiellen Räume bevorzugen. Die bisherige Annahme, daß in diesen nur unter pathologischen Bedingungen sich entfaltenden Spalten und Räumen, die gehirnwärts von der Limitans gliae abgegrenzt werden, Lymphe im engeren Sinne des Wortes zirkuliere, wird in der Arbeit von F. H. Lew $\mathrm{y}^{3}$ ) zurückgewiesen und ausgeführt, daß es sich vielmehr um Kammern handle, zwischen denen ein Austausch flüssiger oder fester Stoffe nur durch Diffusion möglich ist. Immerhin würde die Gewebsflüssigkeit noch wesentlich günstigere Lebensbedingungen schaffen als z. B. das strömende Blut.

1) Neurol. Centralbl. 3 u. 4. 1907.

2) Zieglers Beiträge z. allg. Pathol. u. pathol. Anat. 56. 1913.

$\left.{ }^{3}\right)$ Virchows Archiv, s, folgende Arbeit. 
Allerdings darf dieser aktive, den Protozoen zugeschriebene Faktor nicht überschätzt werden, sondern es muß außerdem noch ein anderes Moment für die Verschiedenartigkeit der Infektionswirkung in Betracht gezogen werden, nämlich die biologischen Abwehrmaßnahmen des erkrankten Körpers. Auch Hauptmann, der im allgemeinen das biologische Verhalten der Spirochäten in den Vordergrund stellt, meint, daß zur Beurteilung des Lues-Metaluesproblems, d.h. dem Bestehen einer reinen mesodermalen Gefäßlues bei Erhaltensein der Limitans gliae gegenüber der ektodermalen Parenchymerkrankung nach Durchbrechung der gliösen Grenzhäute, die Reaktionsmöglichkeit des Organismus herangezogen werden sollte. In diesem Sinne äußert Jakob, daß er in der Paralyse ,,einen ungenügenden und untauglichen Versuch des Körpers, sich der Spirochäten zu entledigen", sehe.

Schon die Beobachtung, daß bestimmte Organe bzw. Organsysteme mit einér gewissen RegelmäBigkeit in ganz bestimmter Reihenfolge erkranken, läßt daran denken, daß in den verschiedenen Organen eine zeitlich und örtlich unterschiedene Immunität des Gewebes für diese Tatsache maßgebend sei, deren Gesetze wir noch nicht kennen. Dafür spricht auch der allgemeine Umstand, daß das Blut eines syphilitisch Infizierten nach einem gewissen Zeitpunkt zunehmend Sterilität erlangt, so daß es nicht mehr infektiös wirkt, analog dem Verhalten bei Schlafkrankheit und multipler Skklerose. Die Krankheitserreger sind also aus dem Blut versehwunden, d. h. durch dort herrschende immunisatorische Vorgänge zugrunde gegangen, während sich gleichzeitig in anderen Körpergeweben Spirochäten ansiedeln und dort krankhafte Veränderungen verursachen können. Besonders bevorzugt für eine solche Spätlokalisation scheint das Zentralnervensystem zu sein, sowohl in seinen Gefäßen, wie in seiner nervösen Struktur. Und man muß annehmen, daß das Nervengewebe eine temporär verschiedene Widerstandsfähigkeit gegen das Krankheitsvirus besitzt, da es lange Zeit hindurch von den dort haftenden Spirochäten nicht geschädigt wird und erst relativ spät seine Widerstandskraft einbüßt, so daß es dann zu Gefäßerkrankungen, paralytischen und tabischen $\mathrm{Zu}$ ständen kommt. Für diese veränderte Reaktionsfähigkeit des Zentralnervensystems werden auch als auslösendes Moment die Allergieverhältnisse angeführt, die bei spezifischer Frühbehandlung durch die nur teilweise erfolgende Immunisierung des Körpers ausgelöst werden (Rezidivstämme).

Für die Klassifizierung solcher Fälle von ausschlaggebender Bedeutung ist das Verhalten der gliösen Grenzhäute. Diesem Punkte wurde bei der Untersuchung große Aufmerksamkeit geschenkt, doch fand sich an keinem der erkrankten bzw. gewucherten Gefäße eine Schädigung dieser gliösen Grenzlamelle, sondern sie war überall erhalten 
geblieben und die Erkrankung so auf den mesodermalen Anteil der Hirnsubstanz beschränkt. Im Gegensatz dazu kommt es zu paralytischen Zustandsbildern, d. h. zu einer ,,parenchymatösen Erkrankung des Gewebes, die nicht nur die Folge schlechter Ernährung durch die Gefäßerkrankung ist nur, nachdem die Grenzmembran für die auftretende Schädigung durchgängig geworden ist".

Daraus ergäbe sich dann die Folgerung, daß für unsern und entsprechende Fälle von sog. Endarteriitis der kleinen Hirngefäße mit Bezug auf diese pathologische Veränderung die Vorbedingung fehlt, um sie den metaluetischen Erkrankungen zuzurechnen.

Damit rückt die Endarteriitis aber in die Reihe der luetischen Gefäßveränderungen, und es ist zu erwägen, ob sie in höherem Grade als die schon besprochene Endarteriitis obliterans von He ubner Anspruch auf das Affix ,,-itis" hat.

Nach der Auffassung Nissls ist die Endarteritis der kleinen Hirngefäße als eine nicht entzündliche Erkrankung anzusehen. Es soll an dieser Stelle nicht etwa die ganze Entzündungsfrage aufgerollt werden. Ich möchte nur einige Bemerkungen anknüpfen, die die Unklarheit beleuchten sollen, die gerade über die Einreihung dieses Prozesses herrscht. Jakob stellt die Befunde bei der Endarteriitis nicht mit Unrecht in Parallele zu den proliferativen Zuständen, wie sie nach Blei- und Salvarsanschädigungen auftreten, und spricht gerade auf Grund der fehlenden Spirochätenbefunde die Vermutung aus, daß hier eine toxische Wirkung vorliegen möchte. Schon mit diesem Vergleich, der in der Tat außerordentlich naheliegt und sich auch bereits in der Bezeichnung als Endarteriitis kundgibt, hat er den Widerspruch aufgewiesen, der sich aus dem nicht entzündlich syphilitischen Gehirnprozeß, der aber weiter als ,,itis" bezeichnet wird, im Gegensatz zu der wenigstens teilweise entzündlichen Paralyse ergibt. Jakob hat das auch offenbar selbst empfunden. wenn er bald nach der obigen Auseinandersetzung die ,innige Verwandtschaft der nicht entzündlichen und entzündlichen Formen der Syphilis des Zentralnervensystems" betont.

Klarfeld ${ }^{1}$ ) hat jüngst kurz darauf hingewiesen, daß mit Rücksicht auf Befunde wie die hier vorliegenden die Möglichkeit zu diskutieren wäre, ob nicht am Gefäßapparat entzündliche Prozesse ohne Infiltrate vorkommen könnten. Gerade auch das Ineinandergreifen infiltrativer und rein proliferativer Gefäßprozesse bei den Schwermetallvergiftungen gibt doch hier zu denken. Bei der Paralyse wie bei der ihr pathologisch nahestehenden Staupe sehen wir in geeigneten Fällen gleichzeitig das Auftreten von stark infiltrierten Gefäßen und von reinen Gefäßneubildungen und -sprossen. Annehmen zu wollen. daß letztere auf toxischem Wege zustandekommen, erstere durch die Spirochäten

\footnotetext{
1) Zeitschr. f. d. ges. Neurol. u. Psychiatr. 58. 1920.
} 
selbst hervorgerufen werden, entbehrt vorläufig noch einer festen Grundlage.

Vielmehr hat Ha utma n n gerade gezeigt, daß die auffallend geringgradige Spirochätendurchsetzung der Gefäße bei der Paralyse in gar keinem Verhältnis zu der Stärke der Infiltration steht. Nur für ein bestimmtes Stadium der Lues scheint die rein mesodermale Verteilung auch im Gehirn zuzutreffen. Es sind bisher nur ganz wenige Fälle von Hirnlues im sekundären Stadium auf Spirochäten untersucht worden. In diesen Fällen hat es sich gezeigt, daß sich gelegentlich Spirochäten auch bereits innerhalb des Nervengewebes finden können. Allerdings ist damit noch gar nichts bewiesen, denn wir wissen ja überhaupt nicht, wann eigentlich bei der Paralyse die Spirochäten in das Hirngewebe einwandern. Hier liegt erstens die Möglichkeit vor, daß die Spirochäten erst in einem späten Stadium die undicht gewordenen Grenzscheiden passieren können. Eine andere Möglichkeit wäre, daß die Infektion bereits sehr frühzeitig vor sich ginge, eine Anschauung, zu deren Stütze man die Gennerichschen Befunde bei systematischer Liquoruntersuchung im Sekundärstadium heranziehen könnte, daß sich aber die klinischen Symptome erst zeigen, nachdem das Gehirn einen verbältnismäßig schweren Grad der Schädigung erlitten hat. Schließlich wäre auch noch zu erwägen, ob die Spirochäten $u$. U. nicht jedes Gehirn primär befallen können, unter normalen Bedingungen aber durch die Abwehrreaktion des Organes gebunden bzw. abgetötet werden und nur zur Wirkung kommen können, wenn irgendwann und aus irgendwelchen Gründen die normale Abwehrreaktion in Wegfall kommt. Als solche Gründe könnten z. B. ungewöhnliche körperliche und geistige Anstrengungen in Frage lzommen, wie ja bekannt ist, daß viele Jahre stationär gebliebene Paralysen nach außerordentlichen Strapazen oder Aufregungen nicht nur einen neuen Schub bekommen, sondern nicht selten in verhältnismäßig kurzer Zeit tödlich endigen können.

Die Frage nach dem Auftreten der Paralyse nach Spirochäteninfektion wird ja nach zwei Seiten hin beurteilt, einerseits nach der Eigenart der Spirochäten, andererseits nach den biologischen Bedingungen im Befallenen. Schon seit langem haben erfahrene Syphilidologen und Praktiker auf die konjugale Tabes und Paralyse hingewiesen und auf die nicht zu kleine Anzahl von Fällen, die nach Infektion aus nachweislich gleicher Quelle sämtlich an Tabes oder Paralyse nach einigen Jahren erkrankt sind. In neuester Zeit hat diese Anschauung durch die von Levaditi ${ }^{1}$ ) vertretene, von P. Marie bekämpfte Lehre von der neurotropen Variante der Spirochäte pallida eine neue Stütze bekommen, wie auch durch die Angaben von Pagnier ${ }^{2}$ ), daß bei der Utbertragung von

1) Compt. rerid. de l'Acad. des Sc. 1920.

2) Presse méd. Mai 1920. 
Paralysespirochäten auf Kaninohen eine längere Inқubation und ein milderer Ablauf zu beobachten sei, und daß die Reimplantation für den Menschen keine Hautpathogenität mehr ergeben hat.

Die Úberleitung von dieser Betrachtungsweise, die die Ursache für die nervöse Nacherkrankung primär in die Spirochäte verlegt, zu jener anderen, die nur die Abwehrreaktion des kranken Organismus für wichtig hält, bilden jene Befunde, die die Möglichkeit einer Spirochätenveränderung im Sinne einer Mutation durch Anpassung offen lassen. Es ist z. B. bekannt, daß in Bosnien und der Herzegowina, wo die Syphilis völlig endemisch wie bei uns die Masern herrscht, der Ausbruch einer Paralyse oder einer Tabes unter der angesessenen Bevölkerung zu den größten Raritäten gehört. Man könnte sich vorstellen, daß durch die lange Durchseuchung eine gewisse Abschwächung oder Umstellung der Virulenz eingetreten ist. Diese Annahme hat wenig Wahrscheinlichkeit für sich, denn sie würde den Schluß in sich bergen, daß die in der angegebenen Weise veränderten Spirochäten auch für die andern unter denselben Bedingungen lebenden Menschen die gleiche Wirkung entfalten. Das ist tatsächlich nicht der Fall. Wir sehen vielmehr, daß die Rasse des Infizierten den Ausschlag gibt. Wir wissen, daß die Neger, die ja zur Lues ein enormes Kontingent stellen, nicht nur in ihrer Heimat, wo sie vielleicht nicht genügend sachverständig beobachtet werden, sondern auch in den Vereinigten Staaten bisher ganz auffallend von der Paralyse verschont gebiieben sind. Rüdin ${ }^{1}$ ) konnte an den Berberstämmen Nordafrikas die Seltenheit der Paralyse gegenüber den unter gleichen Bedingungen mit ihnen lebenden anderen Völkerschaften zeigen. Auch aus Indien liegen ähnliche Beobachtungen vor, so daß Gärtner2) erst neuerdings wieder den Einfluß der höheren Kultur auf das häufigere Auftreten der Paralyse betonte. Gerade wenn wir die Erscheinungsform der Syphilis bei gewissen Naturvölkern berüoksichtigen und, sehen, daß bei ihnen die schwere Eingeweidesyphilis fast gänzlich und sogar die Exantheme z. T. fehlen, wie es Bärmann für die Javaner mitgeteilt hat, so kann man sich des Eindrucks nicht erwehren, daß ein gewisser Zusammenhang zwischen der Schwere der luetischen und dem Auftreten metaluetischer Erkrankungen besteht, die in der anders gearteten Abwehrreaktion der Befallenen gesucht werden muß.

Es wird möglicherweise der Wirklichkeit am nächsten kommen, wenn Hauptmann annimmt, daß die Paralysespirochäte tatsächlich eine biologische Eigenart besitzt, daß dieselbe aber erst durch die abweichenden Abwehrmechanismen des befallenen Organismus hervorgerufen werde.

1) Neurol. Centralbl. 21. 1910.

2) Dtsch. med. Wochenschr. 42. 1920. 
Betrachten wir die Lehren, wie sie sich aus den Beobachtungen über sekundäre Autoinfektionen ergeben, wie sie von Kuczynski an Fleckfieberherden und neuerdings wieder von F. H. Lew $\mathrm{y}$ und Tiefenbach ${ }^{1}$ ) bei Manganperoxydvergiftungen festgestellt worden sind, so liegt die Vermutung nahe, daß durch die zirkulierenden Spirochäten oder ihre Toxine unter besonderen biologischen Verhältnissen derselben und des befallenen Organismus bzw. seiner einzelnen Organe es zu lokalen Schädigungen vielleicht des Nervenparenchyms, vielleicht der Gefäßwandungen im weiteren Sinne direkt kommt, deren Folge u. U. die Durchtrittsmöglichkeit für lebende Spirochäten in das Hirngewebe ist. Erst nach erfolgtem Durchtritt wird es sich dann entscheiden, ob die weitere Ausbreitung und Vermehrung der Spirochäten im Nervengewebe durch vorhandene oder schnell gebildete Abwehrstoffe gehemmt wird oder nicht.

Jedenfalls besteht auch heute noch die Nisslsche Anschauung zu Recht, daß die Intima piae und Limitans gliae den Grenzschutz des Hirngewebes gegen alle Infektionen bilden und daß diese erst nach seiner Durchbrechung das Parenchym befallen können. In diesem Sinne kann man die biologischen Gefäßscheiden als Grenzfaktor zwischen luetischen und metaluetischen Prozessen im Zentralnervensystem bezeichnen. Dann kann man die sog. Endarteriitis der kleinen Hirngefäße aber auch nicht als metaluetisch betrachten, und da sie nach Nissls eigener Definition auch nicht entzündlicher Natur ist, so sollte man bis zur Klärung des Zusammenhanges der infiltrativen und der nicht infiltrativen Gefäßveränderungen einfach von Gefäßneubildungen und -sprossungen oder von Intimaproliferationen sprechen und das Wort Arteriitis für die nach Benda einzig hierhergehörigen Vorgänge aufsparen, die Baumgartensche gummöse Peri- oder Panarteriitis.

Für die Überlassung des Materials, die Anregung und Anleitung zu dieser Arbeit möchte ich auch an dieser Stelle Herrn Dr. F. H. Lew y meinen besten Dank erstatten.

\section{Zusammenfassung:}

Klinische und histologische Beschreibung eines Falles von Tabes, der unter Auftreten psychischer Erscheinungen und bulbärer Lähmungen unter dor Fehldiagnose Paralyse ziemlich schnell zum Tode führte. Die Sektion ergab eine Endarteriit. Iuet. der kleinen Hirnrindengefäße sowie ein großes Aneurysma der Art. bas. mit sekundärer Erweichung im Pons.

Es wird die Beziehung der Heubnerschen Endarteriit. luet. der basalen Hirngefäße zu der der kleinen Hirnrindengefäße besprochen,

1) Zeitschr. f. d. ges, Neurol. u. Psychiatr. 1921.

Virchows Archiv. Bd. 232. 
die engen Beziehungen zwischen beiden dargelegt, und die Endothelwucherung als Reaktion auf die luetische Panarteriitis angesehen.

Es wird die Beteiligung des luet. Prozesses und einer arteriosklerotischen Komponente bei Entstehung des Aneurysmas erörtert.

Im Anschluß an auch in diesem Fall beobachtetes völliges Fehlen des Spirochäten in den Gefäßen werden die verschiedenen Möglichkeiten erwogen, die das Eindringen von Spirochäten in die Hirnrinde, das Entstehen entzündlicher und rein degenerativer Prozesse begünstigen oder veranlassen können. 